\title{
Spotlight on daytime napping during early childhood
}

\author{
Klára Horváth ${ }^{1}$ \\ Kim Plunkett ${ }^{2}$ \\ '2nd Department of Pediatrics, \\ Semmelweis University, Budapest, \\ Hungary; ${ }^{2}$ Department of \\ Experimental Psychology, University \\ of Oxford, Oxford, UK
}

This article was published in the following Dove Press journal: Nature and Science of Sleep
Correspondence: Klára Horváth 2nd Department of Pediatrics, Semmelweis University, Túzoltó utca 7-9, 1094 Budapest, Hungary

Tel +36 I 2I5 I380

Email horvath.klara@med.semmelweisuniv.hu

\begin{abstract}
Daytime napping undergoes a remarkable change in early childhood, and research regarding its relationship to cognitive development has recently accelerated. In this review, we summarize our current understanding of this relationship focusing on children aged $<5$ years. First, we evaluate different studies on the basis of the experimental design used and the specific cognitive processes they investigate. Second, we analyze how the napping status of children may modulate the relationship between learning and napping. Third, the possible role of sleep spindles, ie, specific electroencephalographic components during sleep, in cognitive development is explored. We conclude that daytime napping is crucial in early memory development. Keywords: napping, children, infants, cognitive development, daytime sleep, memory, language development, sleep spindles
\end{abstract}

\section{Introduction}

While the function of nighttime sleep has attracted a great deal of public and scientific scrutiny, curiosity about the function of daytime napping has only recently begun to emerge. This curiosity parallels the increasing interest in infant sleep, as daytime napping contributes significantly to infants' overall sleep time and undergoes rapid change during the first or second year of life. In this review, we summarize our current knowledge about the effects of daytime napping on cognitive functioning in the infant, toddler, and preschooler (children younger than 5 years), focusing on the differences between experimental designs and napping status of children.

In a recent review, Faraut et al outlined our current understanding about the functions of daytime napping and suggested that daytime naps can be a countermeasure for the harmful effects of sleep debt. ${ }^{1}$ Daytime naps have been related to cognitive functions, emotional processing, and self-regulation, as well as health-related factors such as the immune, neuroendocrine, and cardiovascular systems. However, data collected from children are scarce and are mostly concerned with cognition. Going beyond correlational studies investigating the relationship between naps and general cognition, in the past few years, experiments have also been carried out investigating specific cognitive functions, such as memory processes. In a systematic review, Thorpe et al summarized 26 articles about the effects of napping time and quality on development and health in young children. They found the results to be inconsistent most probably because of the different age-groups involved and the different napping statuses of the children studied. ${ }^{2}$ In their review, the authors combined the findings from both correlational 
and experimental studies to draw conclusions. Integrating these methodologies is prone to result in contradictory findings, since correlational studies are often confounded with effects of maturation. Our goal in this article is to update this review with more recent studies, focusing on the cognitive processes tested and the napping status of the infants/children. In addition, we review the few studies which have examined electroencephalographic (EEG) associations.

\section{Development and physiology of daytime naps}

Current understanding of the physiology and regulatory processes underlying napping in early childhood is rather limited, even though daytime naps make up a substantial proportion of total sleep time. It is clear that total sleep time decreases with age, and the reduction is a consequence of less daytime sleep. ${ }^{3,4}$ Furthermore, sleep gradually becomes monophasic during early childhood. ${ }^{5}$ However, the regulatory processes which drive these changes are unknown. In a meta-analysis of observational studies of children aged between 2 months and 12 years, a clear reduction in sleep duration, the number of night awakenings, and the number of daytime naps with age is noticeable; 0 - to 2-year-olds have 1.7 naps per day on average $(\mathrm{SD}=0.6)$. The duration of daytime naps decreases from 3.5 hours ( 1 month) to about 1 hour ( 2 years). ${ }^{3}$ Interestingly, while there are cross-cultural differences in nighttime sleep between predominantly Asian and predominantly Caucasian countries, daytime sleep patterns seem to be more or less universal. In Asian countries, children have shorter nighttime sleep duration and later bed times. However, there are no substantial differences in the number of naps or the duration of daytime sleep. ${ }^{6}$ Thus, the decreasing duration of daytime naps seems to proceed hand in hand with development.

We know much less about the development of sleep architecture of daytime naps compared with nighttime sleep. In an elegant longitudinal design, although on a small sample size, Kurth et al investigated how the structure of naps changes with different amounts of prior wakefulness. ${ }^{7}$ Children were studied at 2, 3, and 5 years, and at each age, they had a nap opportunity at varied times of the day, resulting in varied sleep pressure. Compared with adults, the main difference in preschoolers' daytime sleep composition was the higher proportion of slow-wave sleep and reduced stage 2 sleep. However, while there was a clear reduction in daytime sleep duration across the age-groups involved, the architecture of naps did not differ. Importantly, in older children, there was no increase in slow-wave activity with increasing sleep pressure, indicating that children need less sleep depth during daytime naps as they mature. In other words, there is an attenuated homeostatic drive for increased sleep pressure. One of the reasons for this, proposed by the authors, is that older children exhibit a smaller degree of neural recruitment during wakefulness. ${ }^{8}$ Thus, it is possible that children stop having naps when brain maturity arrives at a point where less neural recruitment is necessary for optimal functioning - when some domain-specific specialization occurred. ${ }^{9}$

\section{Naps and cognition}

It is well established that sleep plays an important role in cognitive functioning in adults, and an increasing number of studies carried out with children support this conclusion. However, in early childhood, naps seem to be at least as important as nighttime sleep. Longitudinal studies indicate a relationship between daytime naps and later cognitive measures, although results are conflicting. ${ }^{10-12}$ Experimental studies help to clarify these results most probably through controlling for the confounding effects of maturation. They suggest that napping influences cognitive functioning such as memory consolidation ${ }^{13-17}$ and abstraction and generalization of learnt information. ${ }^{16,18-20}$ First, we review some correlational and longitudinal studies; then, experimental studies are summarized in three groups: studies investigating declarative memories, statistical learning and generalization, and specifically word learning and generalization of word meanings. Within each category, studies are ordered by age.

\section{Correlational and longitudinal studies}

Correlational studies can shed light on how experimental findings are related to individual differences, whereas longitudinal designs are invaluable for examining predictive relationships. One correlational study was carried out in preschoolers using actigraphy with cognitive testing. A negative relationship was found between the duration of daytime naps and vocabulary size as well as number recall in preschoolers (3- to 5-year-olds), whereas vocabulary and nighttime sleep duration correlated positively. ${ }^{11}$ Unfortunately, the study did not report on the number of days in which the naps occurred during the 7-day monitoring period, making it difficult to distinguish between habitual and nonhabitual nappers.

Longitudinal studies appear inconclusive regarding the napping-cognitive development association. Two studies point toward a negative predictive relationship between napping and cognitive as well as vocabulary development, while one study showed a positive association.

The proportion of daytime and nighttime sleep was investigated in two studies. Bernier et al found that 12-month-old 
infants who sleep more during the night compared with the day have larger expressive vocabulary at 26 months. ${ }^{21}$ Dionne et al found a similar relationship, measuring sleep at 6 and 18 months of age and vocabulary at 18,30 , and 60 months. No relationship between nighttime sleep and vocabulary development was reported. ${ }^{12}$ However, recently, Horváth and Plunkett have reported contrasting results. In this latter study, due to the more powerful statistical analyses used, a greater emphasis was placed on individual differences than on maturational changes. Using growth curve modeling, the authors studied how the patterns of sleep at different times of the day influence the growth of individual vocabulary sizes in terms of both the initial starting point and the pace of development. The study revealed that more frequent daytime naps were positively related to the rate of both expressive (marginal relationship) and receptive vocabulary growth (significant relationship). Interestingly, shorter nighttime sleep duration was associated with larger expressive vocabulary development. ${ }^{10}$ However, the negative effect of nighttime sleep is somewhat counterintuitive. In younger infants, it has been shown that shorter nighttime sleep predicts more advanced mental development, ${ }^{22-24}$ an association which may be the result of a more mature neural system. ${ }^{22,24}$ Others have proposed that longer sleep duration may be the consequence of higher stress reactivity and greater vulnerability. ${ }^{23}$

This pattern of findings suggested that nighttime sleep and daytime naps are of differing importance in vocabulary consolidation, and their relative influence might change with development. Given that there are significant differences in the study designs and the statistical analysis used in the studies reported here, direct comparison presents a number of problems. Hence, further research on the topic is warranted, with systematic investigation of different age-groups - and preferably with the addition of longitudinal examination of sleep patterns.

\section{Experimental studies \\ Declarative memory}

The beneficial impact of naps on declarative memories has been found in both infants and preschoolers. The youngest age-group for which the beneficial effect of a period of sleep on memory consolidation has been reported is 3-montholds. ${ }^{25}$ Infants were habituated to pictures of cartoon figures and tested using the visual paired comparison paradigm after having a nap or staying awake. Only those infants who napped between habituation and test showed a novelty preference, ie, remembered the previously seen stimulus. Unfortunately, to avoid habituation to the distractor stimuli, this design did not provide information on memory immediately after learning. Since novelty preference was observed only in the first trial of testing, which was most probably the result of rapid habituation, it may be concluded that the lack of any novelty preference in the second test trial indicated that memories were unstable against interference. Similarly, fragile memories have been found in 6.5-month-olds by Simon et al, which is reported later in detail. ${ }^{20}$

In 6- and 12-month-old infants, Seehagen et al used the deferred imitation task for three target actions with a puppet, to assess declarative memory consolidation. After learning, infants were tested in a 4-hour and a 24-hour delay in two separate experiments. One group had a nap in the 4-hour retention period, while the other group stayed awake. In addition, there was a baseline condition in which infants did not see the demonstrations of the target actions. In both experiments, only those infants who napped within 4 hours of the demonstration remembered and successfully produced the target actions. There was no significant effect of age, indicating that 6- and 12-month-olds benefit equally from napping. ${ }^{15}$ These results support the idea that frequent naps are necessary in order to be able to efficiently consolidate memories in infancy.

Berger and Scher studied the impact of naps on locomotor problem solving in 13-month-olds. ${ }^{26}$ Toddlers had to crawl through a tunnel to reach the caregiver. This task was challenging for them because the test was scheduled at a time when children have just started walking - at this point, they are reluctant to crawl again. There were different steps in the test which helped the children (eg, placing them on their hands and knees and rolling toys through the tunnel). After a nap, the nap group needed fewer cues to solve the task, while the wake group required the same amount of cues after the same amount of time being awake. Note that this task did not only require declarative memory consolidation, but most probably nondeclarative systems were also recruited.

For preschoolers, similar results were found by Kurdziel et $\mathrm{a}^{14}$ and Giganti et $\mathrm{al}^{27}$ in a within-subject design. In the former, 3- to 5-year-olds were trained in the morning on a visuospatial task in which children had to remember the location of pictures. Recall was tested immediately after training for both nap and wake conditions, and there was no initial difference between the conditions. The second testing took place in the afternoon, after the children in the nap condition had an afternoon nap (children in the wake condition were awake during this period). Performance in the nap condition was significantly better than in the wake condition, as the wake condition's performance declined. Children were also 
tested in the morning of the following day, and in both conditions, performance remained around the same level as in the second testing, indicating that the nap condition's better recall was long-lasting and the nighttime sleep could not restore the performance of the children in wake condition. ${ }^{14}$ In the second study, during the study phase, 40 pictures of objects and animals were presented. To test recognition memory, during the test phase, 20 pictures of the study phase and 20 completely new pictures were presented. Children after a nap showed significantly better performance than after staying awake. However, perceptual priming seemed not to benefit from a short daytime sleep period. ${ }^{27}$

\section{Statistical learning and generalization}

Statistical word segmentation is enhanced after a nap in 6.5-month-olds. ${ }^{20}$ Infants were exposed to an artificial language consisting of four bisyllabic novel words. The transitional probability of the second syllable following the first was 1 for words. Retention was tested in a head-turn preference paradigm using the repetitions of words or partwords (consisting of the previously heard syllables for which the transitional probability was $<1$ ). No-nap infants were matched to nap infants by the duration between training and testing. Only nap infants showed a weak sign of memory retention, indexed by novelty preference, ie, increased listening time for part-words. However, this effect was only apparent in the first block of testing, ${ }^{20}$ again showing fragile memories in young infants, similar to the results found by Horváth et al. ${ }^{25}$

In 12-month-olds, flexible memory retrieval was shown after a nap with the deferred imitation task. After the demonstration of a series of actions on a puppet by the experimenter, infants either had a nap or stayed awake. In the test session, puppets different in color were used. Infants' performance was compared with those in a baseline condition, in which infants did not participate in the demonstration session. Only infants in the nap group differed significantly from baseline, meaning that only children in the nap group were able to retrieve memories and flexibly use them. ${ }^{17}$

The first experimental results regarding the role of naps in cognitive functioning have been found in 15-month-old infants. Infants were presented with word strings with an underlying rule in which the first word predicted the third. Testing followed after 4-hours using the head-turn preference procedure. After a nap, infants were able to generalize this knowledge to novel, previously unheard stimuli, as they listened longer to sentences which had the same predicting pattern as the first postsleep trial type. In contrast, a wake group listened longer to those sentences which contained the same word string pairs as the training stimuli. ${ }^{19}$ Hupbach et al replicated the results. They also found that this effect was long-lasting, as it was apparent during testing on the next day, but only in the nap group. ${ }^{28}$

\section{Word learning and generalization of word meanings}

In children aged under 5 years, three studies have examined how sleep influences word learning. ${ }^{13,16,29}$ Two of them also tested the generalization of word meanings. ${ }^{16,18}$ Friedrich et $\mathrm{al}^{16}$ conducted two experiments on the effect of napping, assessing picture-word priming effects using event-related potentials (ERPs). In the first study, 9- to 16-month-old infants were trained in one of three conditions: in the consistent object pairing condition, one object always appeared with one label; in the consistent category pairing condition, one label corresponded to more, similar objects which formed a category; in the inconsistent pairing condition, one label corresponded to different objects. In the test session, correct object pairings and incorrect object pairings were presented, as well as correct novel category pairings and incorrect novel category pairings, in which novel category corresponds to a new exemplar of the categories. In the training session, initial learning of specific word meanings was indexed by a late negative component which was not previously described. The authors argued that the high demand of the task could be the reason behind this. For category learning, no effects were found on the ERP, which was taken to indicate the acquisition of the name of the category. Those infants who did not nap after training showed no sign of remembering specific word meanings or the generalization of labels to new category exemplars. In contrast, in infants who napped, the N200-500 component was enhanced in the correct object pairing condition compared with the incorrect object pairing condition, indicating that the novel word meanings had been transferred to long-term memory. However, because similar effects were not observable in the N400-like component, the evidence for semantic representation is equivocal. On the other hand, the N400-like effect was diminished in the correct novel category pairing condition compared with the incorrect novel category pairing condition, indicating integration into new semantic representations. ${ }^{16}$ In the second study, 6- to 8-month-old infants were investigated in a similar design. Infants heard new words as labels for new object categories. Generalization was tested by showing novel category exemplars, while either a correct or an incorrect label was played. Infants were assigned to short- (mean $=30$ minutes) and long-nap (mean $=50$ minutes) groups. The brain responses 
of infants in the long-nap group showed a semantic priming effect (similar to the adult N400 effect) by contrast to the brain responses of the short-nap group that most possibly reflected an audio-visual association. These results indicate that only the nap group formed a semantic representation of the new label in the long-term memory. ${ }^{30}$

These findings were supported by two other studies using a different experimental design with 16-month-olds. ${ }^{13,18}$ Using an intermodal preferential looking task, a significantly increased target preference after a nap was found for newly learnt object-label pairs, whereas the performance of the wake group did not change systematically. The training phase contained both an interactive play session and an on-screen training session to maximize learning. Toddlers were also tested in familiar word trials in both sessions and showed positive naming effects without any group difference, indicating that they understood the task and both the nap and the wake groups had similar levels of alertness. ${ }^{13}$ Using a similar design to investigate the generalization of word meanings, the crucial role of napping was also supported. Toddlers were tested on objects that were similar in shape, but different in color to the training objects. Toddlers were only able to generalize the word meaning to another exemplar of a category if they napped after training, ${ }^{18}$ similarly to the study by Friedrich et al. ${ }^{16}$

Horváth et al also evaluated the relationship between vocabulary size and sleep-dependent consolidation of newly learnt word forms and found a positive correlation between expressive vocabulary and the performance improvement after sleep. No such effects were shown for generalization. An explanation offered for these findings was that sleep has a possible role in the fine-tuning of premotor and motor representations. ${ }^{13}$ As an analogy to bird studies, it was proposed that infants whose motor control and vocabulary production were less developed were less able to benefit from sleep due to less established sensorimotor integration. ${ }^{31-33}$ These results suggested that not only were the novel word-object associations consolidated during sleep, but the effectiveness of the consolidation depended on preexisting networks.

Contradictory results regarding generalization in word learning have been reported, though in a slightly older agegroup: 2.5-year-olds. Children learnt labels for three novel categories of objects, which were presented on different backgrounds. During testing, four objects were shown in a trial, a new exemplar of the novel category, a distractor, a novel, and a familiar object. Children were asked to point to the new exemplar by using the label for the category. In addition to the nap and the wake groups, an immediate testing group was also included. There was no sign of generalization in the immediate testing group or in the nap group (tested 4 hours after training and having a nap), as children performed correctly on $<50 \%$ of the trials. However, those children who did not nap between training and testing were able to generalize the category's label to new exemplars. The authors argued that forgetting of irrelevant information is crucial for generalization and that interference from additional exposure in the wake group may have promoted this forgetting. They also claimed that sleep might have strengthened both the relevant and irrelevant features of the category, leading to impaired generalization. ${ }^{34}$

Gomez and Edgin proposed that these results reflect a developmental shift in memory processes. They argued that only over 18 months of age, when the hippocampus is more mature, can sleep have a facilitating role in the consolidation of hippocampal memories. Therefore, results in 15-montholds reflect nonhippocampus-dependent, cortical memory processes that are less able to code precise information. ${ }^{35}$ However, there are two major arguments against this idea. First, the studies detailed above have shown that sleep has an enhancing role in coding precise information of those aged $<18$ months. ${ }^{15,16,25}$ Second, a more recent study by the same research group has found contradictory results. Sandoval et al studied verb generalization in nonhabitually and habitually napping preschoolers. Two actions were performed by two actors, while the actions were named with pseudowords. In the generalization condition, children saw the same actions performed by different actors. Only those children who napped after learning were able to generalize the new names regardless of whether they napped habitually or not. In a different experiment testing initial encoding, the authors did not find any differences between habitually and nonhabitually napping children. ${ }^{36}$ Taken together, there are more studies supporting the view that generalization is enhanced by napping regardless of age, although a possible replication of the results of Werchan and Gomez ${ }^{34}$ would suggest otherwise. Hence, the developmental shift suggested by Gomez and Edgin $^{35}$ has not been supported by current results.

In addition, Williams and Horst ${ }^{29}$ studied how napping influences learning new words from stories, simulating a more realistic learning environment. Three-year-olds were read stories with two new target words. Half of the children heard one story three times, and the other half heard three different stories. Those children who regularly napped slept in the nursery after training, while nonhabitual nappers stayed awake. Repeated readings of the same stories had an immediate positive impact on word learning after the nap, 
but those in the wake group were able to catch up after 24 hours, presumably because they also slept in that interval. However, in the condition in which different stories were read to the children, the long-lasting positive effect was only significant in the nap group. The authors' interpretation of their results was that during the different stories condition, memory traces were weaker, ${ }^{29}$ and sleep was considered to have a larger impact. ${ }^{37}$

On the basis of studies which measure performance after learning, it seems that a decline in the performance of the wake group is responsible for the differences between nap and wake groups for the consolidation of declarative memories, ${ }^{14,16}$ whereas young children are only able to generalize after a nap. ${ }^{16,18,36}$ Furthermore, these effects are long-lasting as they were apparent the day after as well. ${ }^{14,15,27,29,36}$ Napping either helps to consolidate memories in the long term or protects memories from interference. It appears that in the first few months of life, the younger the infant is, the more fragile their memory, either due to less efficient consolidation during wake or due to less stability against interference. ${ }^{20,25}$ Furthermore, the observed associations regarding generalization suggest that sleep does not just passively consolidate memories, but rather that it does so as an active process - referred to as "memory evolution" by Stickgold and Walker. ${ }^{38}$ With this, irrelevant information is forgotten, and the key features of the category are retained.

\section{Effect of napping status}

Young children vary in their napping status, ie, some children are habitual nappers (have naps almost each day), whereas others have naps occasionally. Thorpe et al claimed that after 2 years of age napping is associated with later night sleep onset and both reduced sleep quality and duration. Thus, napping should not be encouraged after sleep has consolidated to the night. ${ }^{2}$ Only three studies have incorporated napping status into their analyses, although we can assume that studies carried out with younger infants had regularly napping participants. Kurdziel et al analyzed how sleep-dependent benefits in performance differ in habitually and nonhabitually napping preschoolers. Interestingly, only habitually napping children benefited from daytime sleep. The authors argued that in those children who do not nap regularly memories are more stable and less likely to decay. ${ }^{14}$ It is possible that in the less mature brain, more frequent consolidation is necessary due to the smaller capacity of the hippocampal stores and/or the less efficient consolidation during wakefulness. This claim may be particularly pertinent for habitually napping children, as in nonhabitual nappers memories appear to be more stable. ${ }^{14}$
Despite not addressing this question directly, Williams and Horst provided some information on napping status and the capacity to consolidate memories during wake in 3-year-olds. In their design, children assigned to a nap group were regular nappers, while the wake group consisted of nonhabitual nappers. Nonhabitual nappers (the wake group) were not able to consolidate the new words from stories while being awake, whereas habitual nappers showed successful consolidation after a nap. ${ }^{29}$

Sandoval et al specifically investigated whether sleepdependent memory consolidation and generalization show different patterns in habitually and nonhabitually napping preschoolers. There was no difference in the performance of the groups; the results indicated that regardless of usual napping behavior, a period of sleep is necessary for generalizing word meanings. ${ }^{36}$

Other studies that provide information about napping status only studied habitually napping children due to their younger ages, ${ }^{13,18,19}$ including a study that did not find a beneficial effect of napping. ${ }^{34}$ The importance of napping status for memory consolidation and generalization therefore remains unresolved, although the weight of the limited evidence available indicates a beneficial effect of napping, irrespective of napping status.

\section{Associations with EEG components}

In a handful of studies of daytime napping, polysomnography has been used, and EEG components of napping were found to be related to cognitive abilities and performance. In all studies, correlations were found with sleep spindles, although the directions of the relationship differed. Kurdziel et al found a negative correlation between sleep spindle density and test performance immediately after learning. However, sleep spindle density was positively correlated with the change in memory performance after napping. ${ }^{14}$ Slow and fast spindles were not analyzed separately, although the authors highlighted that they were identified at central electrodes (C3) where fast spindles are apparent in adults. In Friedrich et al, EEG power in the sleep spindle frequency range over central and parietal areas was correlated with the parietal N400-like priming effect, but no correlation was found with sleep spindle density, count, or spindle length at $\mathrm{C} 3$. Nor did these authors report an association with the N200-500-like component. ${ }^{16}$ Similarly, in Friedrich et al, the N400-like semantic memory effect was positively correlated with the EEG power in the sleep spindle frequency at C4, P4, and Pz electrodes in the long nap group. ${ }^{30}$ Horváth et al found a significant negative correlation between sleep spindle density at frontal and central sites and the time 
needed for habituation. Those infants who were quicker in processing information had a higher sleep spindle density, suggesting links between general cognitive ability and specific sleep components. ${ }^{25}$ Considering that processing speed and the extent of myelinated white matter in the brain show a similar correlation, ${ }^{39}$ we propose that sleep spindling and myelination may be related. Unfortunately, Simon et al did not analyze sleep spindle variables other than spectral density - which was not correlated with behavioral measures. ${ }^{20}$ These data point to some kind of relationship between sleep spindles and cognitive measures. However, it is not clear to what extent spindles reflect general cognitive abilities or a state-like relationship.

Rapid eye movement (REM) sleep also shows substantial changes through development, especially in the first year. ${ }^{40,41}$ Unfortunately, only limited information is available on how much REM sleep daytime naps contain. In early theories, REM sleep has been related to brain maturation. ${ }^{42}$ However, convincing evidence has not been found. ${ }^{43}$ Nevertheless, as REM sleep has been associated with cognitive processing in adults, future studies should examine whether this association also exists in childhood.

\section{Conclusion}

We have briefly reviewed some experimental, correlational, and longitudinal studies investigating how daytime naps and cognitive processes are related in children younger than 5 years of age. In most of the experimental studies considered, a beneficial or a crucial role for naps was found, particularly with respect to generalization, as well as retention. However, contradictory findings have also been reported. Napping seems to provide an optimal environment for consolidating memories, perhaps because it protects fragile memories from interfering stimuli or makes active consolidation and generalization of information possible for young children. It is clear that daytime naps go through developmental changes and become less important with brain maturity. We suppose that the inconclusive results from longitudinal studies may be due to complexities introduced by the triangular relationship between brain maturity, nap duration-frequency, and cognitive development. We propose that as the brain matures, it requires fewer and eventually no daytime naps, probably because of the reduction in brain activation needed during cognitive processing. On the other hand, individual variability in daytime sleep might confound this relationship. Importantly, on the basis of recently published data, it is not evident that beyond the age of 2 years, or after the child stops napping, naps fail to have beneficial cognitive effects. More studies on this question are needed to be able to identify if and when there is an ideal time to stop napping. Future research should use longitudinal studies to compare the nap-cognition relationship before and after sleep has become monophasic. The use of polysomnography would shed light on how the components of sleep differ between daytime naps and nighttime sleep and change with age.

\section{Disclosure}

The authors report no conflicts of interest in this work.

\section{References}

1. Faraut B, Andrillon T, Vecchierini MF, Leger D. Napping: a public health issue. From epidemiological to laboratory studies. Sleep Med Rev. 2017;35:85-100.

2. Thorpe K, Staton S, Sawyer E, Pattinson C, Haden C, Smith S. Napping, development and health from 0 to 5 years: a systematic review. Arch Dis Child. 2015;100:615-622.

3. Galland BC, Taylor BJ, Elder DE, Herbison P. Normal sleep patterns in infants and children: a systematic review of observational studies. Sleep Med Rev. 2012;16:213-222.

4. Blair PS, Humphreys JS, Gringras P, et al. Childhood sleep duration and associated demographic characteristics in an English cohort. Sleep. 2012;35:353-360.

5. Iglowstein I, Jenni OG, Molinari L, Largo RH. Sleep duration from infancy to adolescence: reference values and generational trends. Pediatrics. 2003;111:302-307.

6. Mindell JA, Sadeh A, Wiegand B, How TH, Goh DY. Cross-cultural differences in infant and toddler sleep. Sleep Med. 2010;11:274-280.

7. Kurth S, Lassonde JM, Pierpoint LA, et al. Development of nap neurophysiology: preliminary insights into sleep regulation in early childhood. J Sleep Res. 2016;25(6):646-654.

8. Vyazovskiy VV, Riedner BA, Cirelli C, Tononi G. Sleep homeostasis and cortical synchronization: II. A local field potential study of sleep slow waves in the rat. Sleep. 2007;30:1631-1642.

9. Karmiloff-Smith A. Embrace complexity! Multiple factors contributing to cognitive, social, and communicative development In: Budwig N, Turiel E, Zelazo PD, editors. New Perspectives on Human Development. Cambridge: Cambridge University Press; 2017:386-401.

10. Horváth K, Plunkett K. Frequent daytime naps predict vocabulary growth in early childhood. J Child Psychol Psychiatry. 2016;57:1008-1017.

11. Lam JC, Mahone EM, Mason T, Scharf SM. The effects of napping on cognitive function in preschoolers. J Dev Behav Pediatr. 2011;32:90-97.

12. Dionne G, Touchette E, Forget-Dubois N. Associations between sleepwake consolidation and language development in early childhood: a longitudinal twin study. Sleep. 2011;34:987-995.

13. Horváth K, Myers K, Foster R, Plunkett K. Napping facilitates word learning in early lexical development. J Sleep Res. 2015;24:503-509.

14. Kurdziel L, Duclos K, Spencer RM. Sleep spindles in midday naps enhance learning in preschool children. Proc Natl Acad Sci U S A. 2013;110:17267-17272.

15. Seehagen S, Konrad C, Herbert JS, Schneider S. Timely sleep facilitates declarative memory consolidation in infants. Proc Natl Acad Sci U S A. 2015;112:1625-1629.

16. Friedrich M, Wilhelm I, Born J, Friederici AD. Generalization of word meanings during infant sleep. Nat Commun. 2015;6:6004.

17. Konrad C, Seehagen S, Schneider S, Herbert JS. Naps promote flexible memory retrieval in 12-month-old infants. Dev Psychobiol. 2016;58:866-874.

18. Horváth K, Liu S, Plunkett K. A daytime nap facilitates generalization of word meanings in young toddlers. Sleep. 2016;39:203-207.

19. Gomez RL, Bootzin RR, Nadel L. Naps promote abstraction in language-learning infants. Psychol Sci. 2006;17:670-674. 
20. Simon KN, Werchan D, Goldstein MR, et al. Sleep confers a benefit for retention of statistical language learning in 6.5 month old infants. Brain Lang. 2017;167:3-12.

21. Bernier A, Carlson SM, Bordeleau S, Carrier J. Relations between physiological and cognitive regulatory systems: infant sleep regulation and subsequent executive functioning. Child Dev. 2010;81: 1739-1752.

22. Anders TF, Keener MA, Kraemer H. Sleep-wake state organization, neonatal assessment and development in premature infants during the first year of life. II. Sleep. 1985;8:193-206.

23. Freudigman KA, Thoman EB. Infant sleep during the first postnatal day: an opportunity for assessment of vulnerability. Pediatrics. 1993;92:373-379.

24. Gertner S, Greenbaum CW, Sadeh A, Dolfin Z, Sirota L, Ben-Nun Y. Sleep-wake patterns in preterm infants and 6 month's home environment: implications for early cognitive development. Early Hum Dev. 2002;68:93-102.

25. Horváth K, Hannon B, Ujma PP, Gombos F, Plunkett K. Memory in 3-month-old infants benefits from a short nap. Dev Sci. Epub 2017 Jul 18.

26. Berger SE, Scher A. Naps improve new walkers' locomotor problem solving. J Exp Child Psychol. 2017;162:292-300.

27. Giganti F, Arzilli C, Conte F, Toselli M, Viggiano MP, Ficca G. The effect of a daytime nap on priming and recognition tasks in preschool children. Sleep. 2014;37:1087-1093.

28. Hupbach A, Gomez RL, Bootzin RR, Nadel L. Nap-dependent learning in infants. Dev Sci. 2009;12:1007-1012.

29. Williams SE, Horst JS. Goodnight book: sleep consolidation improves word learning via storybooks. Front Psychol. 2014;5:184.

30. Friedrich M, Wilhelm I, Molle M, Born J, Friederici AD. The sleeping infant brain anticipates development. Curr Biol. 2017;27:2374-2380.e3.

31. Margoliash D, Schmidt MF. Sleep, off-line processing, and vocal learning. Brain Lang. 2010;115:45-58.
32. Tse D, Langston RF, Kakeyama M, et al. Schemas and memory consolidation. Science. 2007;316:76-82.

33. Wilhelm I, Prehn-Kristensen A, Born J. Sleep-dependent memory consolidation-what can be learnt from children? Neurosci Biobehav Rev. 2012;36:1718-1728.

34. Werchan DM, Gomez RL. Wakefulness (not sleep) promotes generalization of word learning in 2.5-year-old children. Child Dev. 2014;85:429-436.

35. Gomez RL, Edgin JO. Sleep as a window into early neural development: shifts in sleep-dependent learning effects across early childhood. Child Dev Perspect. 2015;9:183-189.

36. Sandoval M, Leclerc JA, Gomez RL. Words to sleep on: naps facilitate verb generalization in habitually and nonhabitually napping preschoolers. Child Dev. 2017;88(5):1615-1628.

37. Diekelmann S, Wilhelm I, Born J. The whats and whens of sleepdependent memory consolidation. Sleep Med Rev. 2009;13:309-321.

38. Stickgold R, Walker MP. Sleep-dependent memory triage: evolving generalization through selective processing. Nature Neurosci. 2013;16:139-145.

39. Chevalier N, Kurth S, Doucette MR, et al. Myelination is associated with processing speed in early childhood: preliminary insights. PLoS One. 2015;10:e0139897.

40. Roffwarg HP, Muzio JN, Dement WC. Ontogenetic development of the human sleep-dream cycle. Science. 1966;152:604-619.

41. Olbrich E, Rusterholz T, LeBourgeois MK, Achermann P. Developmental changes in sleep oscillations during early childhood. Neural Plas. 2017;2017:6160959.

42. Marks GA, Shaffery JP, Oksenberg A, Speciale SG, Roffwarg HP. A functional role for REM sleep in brain maturation. Behav Brain Res. 1995;69:1-11.

43. Garcia-Rill E, Charlesworth A, Heister D, Ye M, Hayar A. The developmental decrease in REM sleep: the role of transmitters and electrical coupling. Sleep. 2008;31:673-690.
Nature and Science of Sleep

\section{Publish your work in this journal}

Nature and Science of Sleep is an international, peer-reviewed, open access journal covering all aspects of sleep science and sleep medicine, including the neurophysiology and functions of sleep, the genetics of sleep, sleep and society, biological rhythms, dreaming, sleep disorders and therapy, and strategies to optimize healthy sleep. The manuscript

\section{Dovepress}

management system is completely online and includes a very quick and fair peer-review system, which is all easy to use. Visit http://www. dovepress.com/testimonials.php to read real quotes from published authors. 